\title{
Bacille Calmette-Guérin (BCG) vaccine and the COVID-19 pandemic: responsible stewardship is needed
}

\author{
H. S. Schaaf, ${ }^{1}$ K. du Preez, ${ }^{1}$ M. Kruger, ${ }^{2}$ R. Solomons, ${ }^{2}$ J. J. Taljaard, ${ }^{3}$ H Rabie, ${ }^{2}$ J. A. Seddon, ${ }^{1,4}$ \\ M. F. Cotton, ${ }^{2}$ M. Tebruegge, ${ }^{5,6}$ N. Curtis, ${ }^{5}$ A. C. Hesseling ${ }^{1}$
}

${ }^{1}$ Desmond Tutu TB Centre, Department of Paediatrics and Child Health, Faculty of Medicine and Health Sciences, Stellenbosch University, Cape Town, South Africa; ${ }^{2}$ Department of Paediatrics and Child Health, Faculty of Medicine and Health Sciences, Stellenbosch University, Cape Town, South Africa; ${ }^{3}$ Department of Internal Medicine, Faculty of Medicine and Health Sciences, Stellenbosch University, Cape Town, South Africa; ${ }^{4}$ Department of Infectious Diseases, Imperial College London, London, UK; ${ }^{5}$ Department of Paediatrics, The University of Melbourne, and Murdoch Children's Research Institute, Royal Children’s Hospital Melbourne, Parkville, VIC, Australia; ${ }^{6}$ Department of Infection, Immunity \& Inflammation, UCL Great Ormond Street Institute of Child Health, University College London, London, UK

Correspondence to: H. Simon Schaaf, Department of Paediatrics and Child Health, Faculty of Medicine and Health Sciences, Stellenbosch University, PO Box 241, Cape Town, 8000, South Africa e-mail: $\underline{\text { hss@sun.ac.za }}$

Running head: BCG vaccine: responsible stewardship

Article submitted 16 April 2020. Article accepted 24 April 2020.

\section{Dear Editor,}

We believe that responsible stewardship of the bacille Calmette-Guérin (BCG) vaccine in the context of the COVID-19 epidemic is urgently needed. Live attenuated BCG is currently the only licensed vaccine to protect against tuberculosis (TB). Neonatal BCG vaccination has proven efficacy in protecting infants and young children against life-threatening disseminated forms of TB, including TB meningitis and miliary TB. ${ }^{1-4}$ Global BCG supply shortages in 
recent years (particularly between 2014 and 2017) have highlighted the critical need to sustain newborn BCG vaccination, especially in settings with high TB and HIV burden. ${ }^{5,6}$ The risk associated with low vaccination coverage is highlighted by the dramatic increase in the incidence of TB meningitis in young children reported in association with BCG stock-outs. ${ }^{7}$ Although manufacturer and supply chain issues are now largely resolved, supply remains fragile due to the limited number of production facilities available globally. ${ }^{8}$

\section{BCG has potential non-specific effects including putative impact on COVID-19}

In addition to protecting against TB (its intended target disease), BCG vaccination modulates the immune response to subsequent infections caused by other pathogensthrough the induction of innate immune memory and heterologous lymphocyte activation, resulting in enhanced cytokine production, macrophage activity, T-cell responses and antibody titres, leading to reduced morbidity and mortality. ${ }^{9}$ These non-specific immunological effects of BCG are used to treat bladder carcinoma in adults. ${ }^{10}$

A recent ecological study (yet to be peer-reviewed), has been widely disseminated in the general and social media, and claims a possible association between BCG vaccination and protection against severe disease and fatal outcome from SARS-CoV-2 infection (i.e., COVID19). ${ }^{11}$ This paper provides interesting circumstantial evidence but does not confirm causality. Limitations in this and similar ecological studies imply that claims suggesting potential protective effects of BCG against COVID-19 need to be interpreted with extreme caution. Potential bias in this ecological analysis includes the selection of countries and the failure to take into account the fact that different countries may be at different stages of the COVID-19 epidemic. The exclusion of countries that appear to contradict the authors' conclusions is unexplained. For example, BCG has not been used in Germany for the past 28 years, but currently has one of the lowest COVID-19-related mortality rates globally. Other high-income countries without universal BCG vaccination include Australia, which also has had a low COVID-19 mortality rate, which calls into question any association. Therefore, the suggestion that BCG protects against COVID-19 remains a hypothesis only, with no rigorous, peerreviewed scientific evidence.

There is a need for research regarding the potential effects of BCG on COVID-19

Several trials are planned to investigate whether BCG may indeed offer protection against COVID-19, including in healthcare workers. Such trials have already started in the Netherlands (BCG-CORONA; NCT04328441); in Australia (the BRACE trial; NCT04327206), where BCG is not routinely given to newborns; and in South Africa (TASK-008-BCG-CORONA), 
where BCG is routinely recommended for all newborns. Outcome measures differ across these trials.

In many high TB burden countries, BCG has routinely been administered to newborns since the 1970s. Therefore, healthcare workers under 50 years of age are likely to have received BCG at birth. In these countries, healthcare workers are also commonly infected with Mycobacterium tuberculosis (i.e., have TB infection). ${ }^{12}$ Under these circumstances, BCG administration may lead to a strong local reaction at the injection site (similar to a strongly positive tuberculin skin test reaction). Unfortunately, as a consequence of the article by Miller et $\mathrm{al}^{11}$ some healthcare workers (and members of the general public) are now requesting revaccination as protection for themselves and vaccination of their non-BCG-vaccinated dependents, especially older children. This is understandable given the fear of COVID-19. In this context, it is essential that we provide clear recommendations on BCG vaccination. The use of BCG for an unproven indication is irresponsible and may deplete BCG stocks for young children, for whom it has been proven to be a lifesaving preventive tool against TB-related morbidity and mortality.

\section{Recommendations for high TB burden settings are required}

The World Health Organization (WHO) recently issued a scientific brief calling for BCG to be used for neonatal BCG vaccination only in high-risk settings and not for the prevention of COVID-19. ${ }^{13}$ Likewise, as the co-authors on this paper - who are paediatricians, public health experts and researchers with many years of experience in TB and BCG - we implore you to protect BCG supplies for young children, for whom the vaccine offers substantial proven benefits. Consequently, we provide a series of recommendations (summarized in the Table).

First, the priority remains for neonatal BCG vaccination to be given to all infants in high TB burden settings. No neonate (unless clinically indicated) should leave a birthing facility without BCG vaccination - this should be documented in the vaccination card or the child's handheld health record. Fair allocation of scarce basic resources such as BCG is crucial, and it is an ethical imperative that the most vulnerable should receive the greatest benefit according to Rawls' theory of justice. ${ }^{14}$ Second, infants under 1 year of age who have not yet received BCG, require a catch-up vaccination at any health facility where BCG is available (unless there is a clear contra-indication), even if they are the only infant to be vaccinated from a vial and there is risk of wastage. ${ }^{1}$ Third, as the risk for TB meningitis and miliary TB is the highest in young children under 3 years of age, catch-up BCG, if missed at birth or thereafter, should be administered to this age group once TB infection has been excluded. This is important because of the severe morbidity and mortality associated with TB meningitis. Fourth, older children (>3 
years) should not routinely receive BCG if missed at birth, although we acknowledge that different countries have different guidance regarding the upper age threshold, and the national level guidance should be followed. Fifth, BCG is ineffective as post-exposure prevention for TB. Following documented exposure to Mycobacterium tuberculosis, it is essential that TB preventive therapy is provided according to WHO and local TB guidelines. Finally, there is currently no compelling evidence, either for or against, that BCG protects individuals from COVID-19. Outside of a clinical trial, healthcare workers (or other individuals) should therefore not receive BCG vaccination for protection against COVID-19. Healthcare workers, many of whom are at high risk of COVID-19 disease, should consider enrolling in trials, including those where BCG is used as an intervention, if feasible. Their participation would generate much needed data regarding any potential benefit or risk of BCG vaccination in the context of COVID-19. Importantly, the BCG vaccines used for such trials should be sourced specifically for clinical research, and not from the limited supply available for children in lowincome settings.

\section{Acknowledgements}

$\mathrm{KdP}$ is supported by the Fogarty International Center of the National Institutes of Health (Bethesda, MD, USA) under Award Number K43TW011006.

The content is solely the responsibility of the authors and does not necessarily represent the official views of the National Institutes of Health.

Conflicts of interests: MFC reports grants from Vakzine Projekt Management GmbH (Hannover, Germany) and Serum Institute of India (Pune, India), outside the submitted work. All other authors have nothing to disclose.

\section{References}

1 BCG vaccines: WHO position paper - February 2018. Wkly Epidemiol Rec 2018; 93(8): 73-96.

2 Thwaites GE, van Toorn R, Schoeman J. Tuberculous meningitis: more questions, still too few answers. Lancet Neurol 2013; 12: 999-1010.

3 Trunz BB, Fine P, Dye C. Effect of BCG vaccination on childhood tuberculous meningitis and miliary tuberculosis worldwide: a meta-analysis and assessment of costeffectiveness. Lancet 2006; 367: 1173-1180. 
4 Abubakar I, Pimpin L, Ariti C, et al. Systematic review and meta-analysis of the current evidence on the duration of protection by bacillus Calmette-Guerin vaccination against tuberculosis. Health Technol Assess 2013; 17: 1-372.

5 Marais BJ, Seddon JA, Detjen AK, et al.; WHO Child TB Subgroup. Interrupted BCG vaccination is a major threat to global child health. Lancet Respir Med 2016; 4; 251253.

6 Kontturi A, Santiago B, Tebruegge M, et al. Ptbnet BCG shortage project collaborators, on behalf of the Paediatric Tuberculosis Network European Trials Group (ptbnet). The impact of Bacille Calmette-Guérin shortage on immunisation practice and policies in Europe-A Paediatric Tuberculosis Network European Trials Group (ptbnet) survey. Tuberculosis (Edinb) 2016; 101: 125-129.

7 du Preez K, Seddon JA, Schaaf HS, et al. Global shortages of BCG vaccine and tuberculous meningitis in children. Lancet Glob Health 2019; 7: e28-e29.

8 Cernuschi T, Malvolti S, Nickels E, et al. Bacillus Calmette-Guerin (BCG) vaccine: a global assessment of demand and supply balance. Vaccine 2018; 36: 498-506.

9 Moorlag S, Arts RJW, van Crevel R, et al. Non-specific effects of BCG vaccine on viral infections. Clin Microbiol Infect 2019; 25: 1473-1478.

10 Alhunaidi O, Zlotta AR. The use of intravesical BCG in urothelial carcinoma of the bladder. Ecancermedicalscience 2019; 13: 905. doi: 10.3332/ecancer.2019.905. eCollection 2019.

11 Miller A, Reandelar MJ, Fasciglione K, et al. Correlation between universal BCG vaccination policy and reduced morbidity and mortality for COVID-19: an epidemiological study. medRxiv 2020; https://doi.org/10.1101/2020.03.24.20042937

12 Grobler L, Mehtar S, Dheda K, et al. The epidemiology of tuberculosis in health care workers in South Africa: a systematic review. BMC Health Serv Res 2016; 16: 416.

13 World Health Organization. Bacille Calmette-Guérin (BCG) vaccination and COVID19. Scientific brief. Geneva, Switzerland: WHO, 2020. https://www.who.int/newsroom/commentaries/detail/bacille-calmette-gu\%C3\%A9rin-(bcg)-vaccination-andcovid-19 Accessed April 2020.

14 Daniels N. Justice, health, and healthcare. Am J Bioeth 2001; 1: 2-16. 
Table Recommendations for BCG vaccination in high TB burden settings

\begin{tabular}{lc}
\hline Priority populations & Further recommendations \\
\hline $\begin{array}{l}\text { Neonatal BCG vaccination remains the } \\
\text { priority }\end{array}$ & $\begin{array}{c}\text { National guidelines regarding the upper age } \\
\text { limit for catch-up vaccination should be } \\
\text { followed }\end{array}$ \\
$\begin{array}{l}\text { Unvaccinated infants should be prioritised } \\
\text { for catch-up vaccination, irrespective of } \\
\text { possible vaccine wastage }\end{array}$ & BCG vaccination should NOT be used for \\
$\begin{array}{l}\text { Children younger than 3 years who have not } \\
\text { been vaccinated, should also be prioritised following TB exposure } \\
\text { to receive catch-up vaccination once TB } \\
\text { infection has been excluded }\end{array}$ & protection against COVID-19 outside of \\
\end{tabular}

TB = tuberculosis; BCG = bacille Calmette-Guérin. 